\title{
Fructose-1,6-bisphosphatase-1 decrease may promote carcinogenesis and chemoresistance in cervical cancer
}

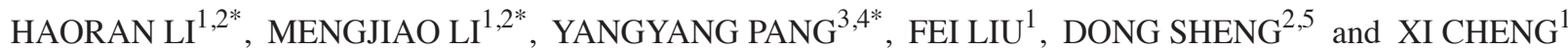 \\ ${ }^{1}$ Department of Gynecologic Oncology, Fudan University Shanghai Cancer Center; ${ }^{2}$ Department of Oncology, \\ Shanghai Medical College, Fudan University, Shanghai 200032; ${ }^{3}$ Institute of Urology, Lanzhou University Second \\ Hospital, Lanzhou University; ${ }^{4}$ Key Laboratory of Urological Diseases, Lanzhou University, Lanzhou, Gansu 730000; \\ ${ }^{5}$ Department of Pathology, Fudan University Shanghai Cancer Center, Shanghai 200032, P.R. China
}

Received March 26, 2017; Accepted September 5, 2017

DOI: $10.3892 / \mathrm{mmr} .2017 .7665$

\begin{abstract}
Fructose-1,6-bisphosphatase-1 (FBP1), a gluconeogenesis rate-limiting enzyme expressed in various tissues, is important in the carcinogenesis of various cancers. To evaluate the association of FBP1 expression and carcinogenesis and chemoresistance in cervical cancer, the present study analyzed 140 patients of squamous cell carcinoma of cervical cancer (CSCC) who had adjuvant concurrent chemoradiation therapy following radical surgery. By detecting FBP1 protein expression in paraffin-embedded tumor tissues through immunohistochemistry, it was observed that $50 \%$ of the cases had a low expression of FBP1, which was associated with a shorter overall survival time $(\mathrm{P}=0.011)$. In addition, $\mathrm{FBP} 1 \mathrm{mRNA}$ level was downregulated in tumor tissues, compared with cervical normal tissues. Among the tumor-associated prognostic factors, loss of FBP1 expression $\left(\chi^{2}\right.$-test, $\left.\mathrm{P}=0.025\right)$ was significantly associated with the tumor recurrence and greater tumor stage of cervical cancer patients (2-test, $\mathrm{P}<0.0001)$. In 3-(4,5)-dime thylthiahiazo(-z-y1)-3,5-diphenytetrazoliumromide (MTT) assay of primary tumor cells, the median in vitro inhibition rate of cisplatin, carboplatin, nedaplatin, and oxaliplatin was $62,47,58$ and $52 \%$, respectively. Although there was no significant association between FBP1 expression and in vitro tumor inhibition rates of primary tumor cells, overexpression of FBP1 markedly suppressed carcinogenesis and restored the chemosensitivity to cisplatin in cervical cancer cell lines of HeLa and CaSki. Overall, decreased levels of FBP1 may be used as a predictor for poor prognosis of cervical cancer
\end{abstract}

Correspondence to: Dr Xi Cheng, Department of Gynecologic Oncology, Fudan University Shanghai Cancer Center, 270 Dongan Road, Shanghai 200032, P.R. China

E-mail: cheng_xi1@hotmail.com

*Contributed equally

Key words: fructose-1,6-bisphosphatase-1, carcinogenesis, chemoresistance, cervical cancer patients, however the mechanism requires further investigation.

\section{Introduction}

Cervical cancer is the second most commonly diagnosed cancer and third leading cause of cancer death among females in developing countries, accounting for an estimated $3.7 \%$ $(527,600)$ new cancer cases and 3.2\% $(265,700)$ deaths worldwide in 2012 (1). Patients with squamous cell carcinoma of cervical cancer (CSCC) who have locally advanced and recurrent and/or metastatic disease are mostly treated with concurrent chemoradiotherapy (CRT) (2-4). Cisplatin-paclitaxel combination is still the standard regimen in CRT compared with other cisplatin-based chemotherapy $(5,6)$. However, the widespread adoption of platinum-based chemoradiotherapy protocols for CSCC patients would make these drugs less effective when acquired resistance occurs.

Fructose-1,6-bisphosphatase-1 (FBP1) is a gluconeogenesis rate-limiting enzyme used to catalyzes the hydrolysis of fructose-1,6-bisphosphate to fructose-6-phosphate and inorganic phosphate. There are two isoenzymes of FBP in mammalian cells, FBP1 and FBP2. FBP1 is widely expressed in different tissues while the expression of FBP2 is limited to muscle $(7,8)$. FBP1 deficiency is associated with hypoglycemia and metabolic acidosis. Interestingly, recent studies have shown that decreased level of FBP1 expression was observed in several solid tumors such as liver, colon, gastric and renal tumors, and was associated with poor prognosis in patients (8-10). Promoter hypermethylation may probably contribute to this phenomenon even though the exact mechanism is still unclear (11). In addition, FBP1 appears to be a functional tumor suppressor involved in the carcinogenesis through inhibiting a potential 'Warburg effect' that cancer cells have a higher rate of aerobic glycolysis compared with oxidative phosphorylation $(12,13)$.

However, the role of FBP1 in regulating the carcinogenesis and chemoresistance in cervical cancer has not been well validated. In the present study, we detected the expression of FBP1 in CSCC patients, and investigated the associations between FBP1 expression and the carcinogenesis as well as chemosensitivity in cervical cancer cell lines. 


\section{Materials and methods}

Compliance with ethical standards. All procedures performed in this study involving human participants were in accordance with the ethical standards of the Institutional Review Board of FUSCC and with the 1964 Helsinki declaration and its later amendments or comparable ethical standards.

Tissue samples and cell lines. We recruited 140 consecutive CSCC patients with International Federation of Gynecology and Obstetrics (FIGO, 2009) stages IB, IIA or IIB, who had radical hysterectomy and pelvic lymphadenectomy with histopathologic confirmed high-risk factors. All patients were treated with adjuvant concurrent chemoradiation therapy at Fudan University Shanghai Cancer Center (FUSCC) between March 2008 and March 2009. The cases were histopathologically confirmed to be squamous cell carcinoma by two gynecologic pathologists. Detailed clinical information has been described elsewhere (14). Patients were followed every 3 months for the first 2 years, every 6 months for the next 2 years, and annually for the following years thereafter. Disease-free survival (DFS) and overall survival (OS) duration were calculated from the date of first surgery to the date of disease recurrence and death or the last follow-up visit, respectively. Patients without progression, lost to follow-up or died from other causes, were censored at their last date of record. The project was approved by the Institutional Review Board of FUSCC. Written informed consents were obtained from all recruited individuals, and each clinical investigation was conducted according to the principles expressed in the Declaration of Helsinki consent.

The established human cervical cancer cell lines HeLa and CaSki were obtained from American Type Culture Collection (ATCC). All cells were maintained in Dulbecco's modified Eagle's medium (DMEM; Hyclone; Thermo Scientific, Waltham, MA, USA) supplemented with $10 \%$ fetal bovine serum (Gibco Life Technologies, Grand Island, NY, USA), $100 \mathrm{U} / \mathrm{ml}$ penicillin, and $100 \mathrm{U} / \mathrm{ml}$ streptomycin (both from Biowest, Nuaillé, France) and incubated at $37^{\circ} \mathrm{C}$ in a humidified atmosphere with $5 \% \mathrm{CO}_{2}$.

Immunohistochemistry (IHC) assay. The 10x12 (120 cores) tissue microarray (TMA) was made by FUSCC Tissue Bank, as described previously (15). Each case has two cores made from separate sources. IHC was performed on $5-\mu \mathrm{m}$-thick TMA sections from formalin-fixed, paraffin-embedded tissue using the antibody against FBP1 (ab196556, mouse polyclonal antibody, 1:200 dilution; Abcam, Cambridge, MA, USA). The positive control is a known positive case sample. And non-immune goat serum takes the place of primary antibody serving as the negative control. The IHC staining results were scored independently by two gynecologic pathologists, who were blinded to clinical information of the patients. Scoring system is on the basis of both percentage of positive tumor cells and staining intensity, as described previously (16). Briefly, staining intensity was graded as 0 (none), $1+$ (weak), $2+$ (intermediate) and 3+ (strong), and percentage of positive tumor cells was graded as $1+(<10 \%$ of the cells), $2+(10-50 \%$ of the cells) or $3+(\geq 50 \%$ of the cells). The combination of intensity and distribution was graded as $0(<1+), 1(1+$ to $2+)$,
$2(>2+$ to $4+)$ or $3(>4+$ to $6+)$. Finally, the assessment of the protein expression was defined as negative (grade 0 or 1 ) and positive (grade 2 or 3 ).

RT-PCR and real-time PCR. Total RNA was isolated from fresh frozen tumor tissues and normal tissues of 20 CSCC patients using TRIzol reagent (Invitrogen Life Technologies, Carlsbad, CA, USA) and reversely transcribed into cDNA using PrimeScript ${ }^{\mathrm{TM}}$ RT Reagent kit (Takara Bio, Inc., Otsu, Japan). PCR products were amplified with Takara $\mathrm{Taq}^{\mathrm{TM}}$ with reactions of 30 cycles of $\left(94^{\circ} \mathrm{C}, 30 \mathrm{sec} ; 58^{\circ} \mathrm{C}, 30 \mathrm{sec}\right.$ and $72^{\circ} \mathrm{C}$, $1 \mathrm{~min}$ ) using the Master Cycler 1 Eprealplex (Eppendorf AG, Hamburg, Germany). PCR products $(5 \mu \mathrm{l})$ were analyzed by electrophoresis on $1.5 \%$ agarose gel containing ethidium bromide and visualized under UV illumination. Real-time PCR was carried out in the Applied Biosystems Prism 7900 system (Applied Biosystems, Life Technologies, Foster City, CA, USA) using ExScipt SYBER-Green QPCR kit (Takara Bio, Inc.) in the following conditions: An initial denaturation of $95^{\circ} \mathrm{C}$ for $30 \mathrm{sec}, 1 \mathrm{cycle} ; 95^{\circ} \mathrm{C}$ for $5 \mathrm{sec} ; 55^{\circ} \mathrm{C}$ for $30 \mathrm{sec}$; and $72^{\circ} \mathrm{C}$ for $30 \mathrm{sec}, 40$ cycles followed by a melting curve analysis to check the specificity of amplification. Each sample was tested in triplicate, and primers to glyceraldehyde 3-phosphate dehydrogenase (GAPDH) were used in parallel reactions as internal control. Three independent experiments were done for final analyses using the $2-\Delta \Delta C \mathrm{C}$ relative quantification method. The primer pairs of FBP1 used were CTACGCCAG GGACTTTGACC and GGCCCCATAAGGAGCTGAAT. The primer pairs of GAPDH were 5'-GGCCTCCAAGGA GTAAGACC-3' (forward primer) and 5'-CAAGGGGTCTAC ATGGCAAC-3' (reverse primer).

Plasmid construction and cell transfection. To selectively overexpress FBP1, the plasmid pENTER-FBP1, containing human full cDNA sequence of FBP1, was purchased from Vigene Biosciences (Jinan, China). The recombinant plasmid PCDH/FBP1 cDNA was generated by subcloning the cDNA sequence of FBP1 into lentivirus vector PCDHCMV- MCSEF1-PURO. Both of HeLa and CaSki cells were transfected with the PCDH/FBP1 cDNA or the PCDH/negative control for a total of 4 days ( 2 days for each infection) and the positive clones were selected with puromycin $(200 \mathrm{ng} / \mathrm{ml})$ for 7-10 days.

Western blot analysis. Western blot analysis was performed to determine the expression levels of FBP1 and glycolysis-related protein GLUT1, GLUT4, LDHB in cervical cancer cell lines. Cells were harvested, washed with cold 1X PBS, and lysed with RIPA lysis buffer (Beyotime Institute of Biotechnology, Haimen, China) for $30 \mathrm{~min}$ on ice, then centrifuged at $12,000 \mathrm{~g}$ for $15 \mathrm{~min}$ at $4^{\circ} \mathrm{C}$. The total protein concentration was determined by BCA Protein Assay kit (Beyotime Institute of Biotechnology). Equal amounts (30 $\mu \mathrm{g}$ per load) of protein samples were subjected to SDS-PAGE electrophoresis and transferred on to polyvinylidene fluoride (PVDF) membranes (Millipore, Billerica, MA, USA). The blots were blocked in $8 \%$ non-fat milk, and incubated with primary antibodies, followed by incubation with secondary antibodies conjugated with horseradish peroxidase (HRP). The protein bands were developed with the chemiluminescent reagents (Millipore). 
Antibody to FBP1 was from Abcam, GLUT1, GLUT4, LDHB were from ProteinTech Group, Inc. (Chicago, IL, USA), and antibody to $\beta$-actin was purchased from Sigma-Aldrich (St. Louis, MO, USA).

Colony formation assay. Cells were seeded in 6-well plates at a density of 500 per well. The fresh medium was added to allow cell growth for at least one week. The colonies with more than 50 cells were counted after staining with gentian violet (Beijing Solarbio Science and Technology Co., Ltd., Beijing, China). Three independent experiments were done in triplicate wells.

Cell proliferation assay. To evaluate cell proliferation rate, we plated $1 \times 10^{3}$ cells per well in 96-wells plates with $100 \mu \mathrm{l}$ maintenance medium. Cell Counting Kit-8 (CCK-8) (Dojindo Laboratories, Kumamoto, Japan) was used to monitor cell growth at 0-7 day and the number of viable cells was assessed by measurement of absorbance at $450 \mathrm{~nm}$ by a Microplate Reader (BioTek Instruments, Inc., Winooski, VT, USA). The proliferation index was calculated as experimental OD value/control $\mathrm{OD}$ value. Cell numbers were calculated with the following equation: Cell number $=$ proliferation index $\times 1,000$.

Cell viability assay. Cell viability was also evaluated by CCK-8. We plated $8 \times 10^{3}$ cervical cancer cells per well in $96-w e l l$ plates. The next day, the cells were treated with various concentrations of cisplatin purchased from Sigma-Aldrich. Cell viability was then measured as described above. Three independent experiments were done in triplicate wells.

We used the 3-(4,5)-dimethylthiahiazo(-z-y1)-3,5-diphenytetrazoliumromide (MTT) assay to detect in vitro inhibition rates of four platinum agents (cisplatin, carboplatin, nedaplatin and oxaliplatin), as described previously (15). Optical densities $\left(\mathrm{OD}_{570 \mathrm{~nm}}\right)$ were measured by Microplate Reader (Bio-Rad 550; Bio-Rad Laboratories, Hercules, CA, USA). The inhibition rate $=\left(1-\mathrm{OD}_{\text {platinum }} / \mathrm{OD}_{\text {control }}\right) \times 100 \%$.

Statistical methods. We performed the Pearson's $\chi^{2}$-test to evaluate differences in the distributions of FBP1 expression with clinical and pathological variables. Kruskal-Wallis test was used to compare tumor inhibition rates among different groups. Kaplan-Meier curve and multivariate Cox proportional hazards regression analysis (including enter, back and forward Wald tests) were conducted for survival estimate. All statistical analyses were performed with SPSS version 19.0 (SPSS, Inc., Chicago, IL, USA). Each reported P-value was two sided with a significance level of $\mathrm{P}<0.05$.

\section{Results}

Loss of FBPI is a negative prognostic factor in CSCC patients. Clinical and pathological characteristics of the 140 CSCC patients enrolled in the study are summarized in Table I (the status of HPV was not included for the reason that most of the patients had not done HPV tests before radical surgery). The median follow-up time was 34.6 months (range, 20.0-40.0 months). There were 24 (17.1\%) recurrences and $10(7.1 \%)$ deaths. The median time to recurrence was 17.1 months. According to the scoring criteria, we observed
$70(50 \%)$ patients with FBP1-positive (Fig. 1A) and $70(50 \%)$ with FBP1-negative (Fig. 1B) expression, respectively. Interestingly, FBP1 protein levels exhibited a prognostic value, as $\mathrm{OS}(\mathrm{P}=0.011)$ and $\mathrm{DFS}(\mathrm{P}=0.026)$ time were markedly shortened in patients whose tumors exhibited low FBP1 protein levels (Fig. 1C and D). By using log-rank test and multivariate Cox proportional hazards regression model (including enter, back and forward Wald tests), we evaluated potentially prognostic factors in CSCC patients (Fig. 1). Univariate analysis showed that patients with lympho-vascular space invasion (LVSI) ( $\mathrm{P}=0.023$; Table IA) and low expression of FBP1 ( $\mathrm{P}=0.011$; Table IA) had shorter OS time. In multivariate analysis, there was no significant associations in enter mode (Table IA); however, both of back and forward Wald tests revealed that low expression of FBP1 was significantly related to prognosis of CSCC patients (Wald=4.470, HR=9.287, 95\% CI=9.287 (1.177-73.312), P=0.034; Table II). We further explored the association of clinicopathological characteristics with recurrence in CSCC patients and found that FBP1 expression $\left(\chi^{2}\right.$-test, $\left.\mathrm{P}=0.025\right)$ was significantly associated with the recurrent status of cervical cancer patients (Table IA). In addition, the expression level of FBP1 had a negative correlation with tumor stage among various prognostic factors of CSCC $\left(\chi^{2}\right.$-test, $\left.\mathrm{P}=0.000\right)$ (Table III).

FBP1 regulates carcinogenesis in cervical cancer. As the loss of FBP1 is a critical oncogenic event in many cancers $(9,11,17)$, we thus investigated the role of FBP1 in human CSCC. We first tested the messenger RNA (mRNA) levels in 20 human CSCC tissues and normal cervical tissues (clinical pathological features; Table IV). The result showed that mRNA expression of FBP1 is lower in human CSCC tumor tissues than normal cervical tissues $(\mathrm{P}=0.0005$; Fig. $1 \mathrm{E})$. To further confirm the role of FBP1 in cancer cell growth inhibition, we established HeLa/FBP1 and CaSki/FBP1 cells stably expressing FBP1 cDNA and then performed cell colony formation assay and proliferation assay. The results of CCK- 8 and colony formation assay exhibited that the ability of cervical cancer cell growth and proliferation was obviously weakened by the induction of FBP1 when compared with controls $(\mathrm{P}<0.05$ or $\mathrm{P}<0.01$; Fig. 2A-D).

Then, we detected the glycolysis-related protein expression in HeLa and CaSki cells with or without FBP1 by western blot analysis. The results exhibited that protein level of GLUT1, GLUT4 and LDHB were downregulated compared with their controls $(\mathrm{P}<0.01$; Fig. 2E), indicating FBP1 was involved in cancer metabolism.

Above all, FBP1 inhibited cancer growth and proliferation in cervical cancer cells by regulating the expression of glycolysis related protein such as GLUT1, GLUT4 and LDHB.

FBP1 overexpression restores chemosensitivity of cervical cancer cells. The MTT assay aims to detect in vitro inhibition rates of four platinum agents (cisplatin, carboplatin, nedaplatin, and oxaliplatin), as described previously (12). The median in vitro inhibition rate of tumor cell growth by cisplatin, carboplatin, nedaplatin, and oxaliplatin was $62,47,58$, and $52 \%$, respectively. Four platinum agents showed a significantly difference in inhibiting CSCC cells (Kruskal-Wallis test, $\mathrm{P}<0.0001)$. Cisplatin and nedaplatin group had larger 
Table I. Association of clinicopathological characteristics with recurrence in CSCC patients.

A, Association of clinicopathological characteristics with recurrence in CSCC patients (in situ)

\begin{tabular}{|c|c|c|c|c|c|c|}
\hline \multirow[b]{2}{*}{ Prognostic factors } & \multirow[b]{2}{*}{$\begin{array}{l}\text { All patients } \\
\text { N }(\%)\end{array}$} & \multirow{2}{*}{$\begin{array}{c}\text { Non recurrence } \\
\mathrm{N}(\%)\end{array}$} & \multirow{2}{*}{$\begin{array}{c}\text { Recurrence } \\
\text { N (\%) }\end{array}$} & \multirow{2}{*}{$\begin{array}{l}\text { Univariate } \\
\text { P-value }^{\mathrm{a}}\end{array}$} & \multicolumn{2}{|c|}{ Multivariate (enter) } \\
\hline & & & & & $\operatorname{HR}(95 \% \mathrm{CI})^{\mathrm{b}}$ & P-value ${ }^{b}$ \\
\hline All patients & $140(100)$ & $116(82.9)$ & $24(17.1)$ & & & \\
\hline Age, years & & & & $0.370^{c}$ & & \\
\hline$\leq 46($ median $)$ & $70(50)$ & $60(85.7)$ & $10(14.3)$ & & 1.000 & \\
\hline$>46$ (median) & $70(50)$ & $56(80)$ & $14(20)$ & 0.935 & $0.943(0.224-3.967)$ & 0.936 \\
\hline FIGO stage & & & & $0.106^{c}$ & & \\
\hline IB & $70(50)$ & $61(87.1)$ & $9(12.9)$ & & 1.000 & \\
\hline IIA & $64(45.7)$ & $52(81.3)$ & $12(18.8)$ & & 1.000 & \\
\hline IIB & $6(4.3)$ & $3(50)$ & $3(50)$ & 0.496 & $0.353(0.030-4.200)$ & 0.410 \\
\hline Tumor size $^{\mathrm{d}}, \mathrm{cm}$ & & & & $0.575^{\mathrm{c}}$ & & \\
\hline$\leq 4$ & $83(59.3)$ & $70(84.3)$ & $13(15.7)$ & & 1.000 & \\
\hline$>4$ & $57(40.7)$ & $46(80.7)$ & $11(19.3)$ & 0.550 & $1.669(0.427-6.524)$ & 0.461 \\
\hline Pelvic LN & & & & $0.595^{\mathrm{c}}$ & & \\
\hline Negative & $94(67.1)$ & $79(84)$ & $15(16)$ & & 1.000 & \\
\hline Positive & $46(32.9)$ & $37(80.4)$ & $9(19.6)$ & 0.227 & $0.415(0.070-2.446)$ & 0.331 \\
\hline LVSI & & & & $0.058^{\mathrm{c}}$ & & \\
\hline Negative & $88(62.9)$ & $77(87.5)$ & $11(12.5)$ & & 1.000 & \\
\hline Positive & $52(37.1)$ & $39(75)$ & $13(25)$ & $0.023^{\mathrm{e}}$ & $6.979(0.933-52.189)$ & 0.058 \\
\hline Depth of cervical stromal invasion & & & & $0.309^{c}$ & & \\
\hline$\leq 2 / 3$ & $83(59.3)$ & $71(85.5)$ & $12(14.5)$ & & 1.000 & \\
\hline$>2 / 3$ & $57(40.7)$ & 45 (78.9) & $12(21.1)$ & 0.157 & $1.679(0.321-8.777)$ & 0.539 \\
\hline P16 expression status & & & & $0.585^{\mathrm{c}}$ & & \\
\hline Negative & $29(20.7)$ & $23(79.3)$ & $6(20.7)$ & & 1.000 & \\
\hline Positive & $111(79.3)$ & $93(83.8)$ & $18(16.2)$ & 0.932 & $1.077(0.183-6.329)$ & 0.934 \\
\hline FBP1 expression status & & & & $0.025^{\mathrm{c}, \mathrm{e}}$ & & \\
\hline Negative & $70(50)$ & $53(75.7)$ & $17(24.3)$ & $0.011^{\mathrm{e}}$ & $8.850(0.957-81.834)$ & 0.055 \\
\hline Positive & $70(50)$ & $63(90)$ & $7(10)$ & & 1.000 & \\
\hline
\end{tabular}

B, Association of clinicopathological characteristics with recurrence in CSCC patients (in vitro)

Multivariate (enter)

Prognostic factors

All patients Non recurrence Recurrence Univariate

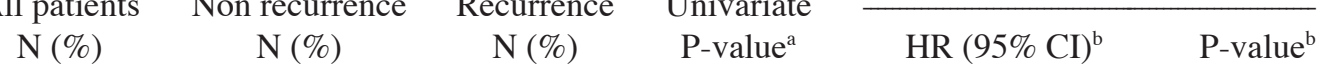

All patients

Cisplatin inhibiton rates

$\leq 0.74$ (median)

$>0.74$ (median)

Carboplatin-inhibition rates

$\leq 0.40$ (median)

$>0.40$ (median)

Nedaplatin-inhibition rates

$\leq 0.71($ median $)$
$>0.71($ median $)$

Oxaliplatin-inhibition rates

$\leq 0.52$ (median)

$>0.52$ (median)

$140(100) \quad 116(82.9) \quad 24(17.1)$

$53(37.9)$

$46(86.8)$

$70(80.5)$

$7(13.2)$

$87(62.1)$

$62(83.8)$

$17(19.5)$

$54(81.8)$

$12(16.2)$

74 (52.9)

66 (47.1)

12 (18.2)

$50(84.7)$

$9(15.3)$

59 (42.1)

$66(81.5)$

15 (18.5)

67 (47.9)

58 (86.6)

$73(52.1)$

$9(13.4)$

15 (20.5) $0.335^{\mathrm{c}}$

0.234

$0.758^{\mathrm{c}}$

0.675

$0.613^{\mathrm{c}}$

$0.269(0.052-1.398)$

1.000

0.118

$0.282(0.049-1.635)$

1.000

0.158

$2.525(0.420-15.178)$

1.000

0.311

$0.264^{\mathrm{c}}$
$4.438(0.716-27.509)$

1.000
0.109

A: ${ }^{a}$ Without adjustment and ${ }^{\mathrm{b}}$ with adjustment (enter) for all of the prognostic factors. ${ }^{\circ}$ Two-sided $\chi^{2}$ test for distributions between patients with non-recurrence and recurrence. ${ }^{\mathrm{d}}$ The largest tumor diameter after surgical resection. B: ${ }^{\mathrm{a}} \mathrm{Log}$-rank test and ${ }^{\mathrm{b}}$ with adjustment (enter) for all of the prognostic factors ${ }^{~}$ Two-sided $\chi^{2}$ test for distributions between patients with non-recurrence and recurrence. ${ }^{e} \mathrm{P}<0.05$. CSCC, squamous cell carcinoma of cervical cancer; FIGO, International Federation of Gynecology and Obstetrics; LN, lymph node; LVSI, lympho-vascular space invasion; FBP1, fructose-1,6-bisphosphatase-1; HR, hazard ratio; CI, confidence interval. 
Table II. Cox proportional hazards regression analysis of CSCC patients (back and forward Wald tests).

\begin{tabular}{lccccr}
\hline $\begin{array}{l}\text { Prognostic factors (with significance) } \\
\text { FBP1 expression status }\end{array}$ & B & SE & Wald & HR & 95\% CI \\
Negative & 2.229 & 1.054 & 4.470 & 9.287 & $1.177-73.312$ \\
Positive & & & & 1.000 & $\mathbf{0 . 0 3 4}$ \\
\hline
\end{tabular}

Values in bold font indicate statistical significance $(\mathrm{P}<0.05)$. CSCC, squamous cell carcinoma of cervical cancer; FBP1, fructose-1,6-bisphosphatase-1; SE, standard error; HR, hazard ratio; CI, confidence interval.

A

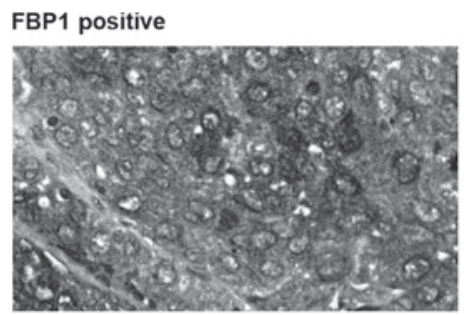

C

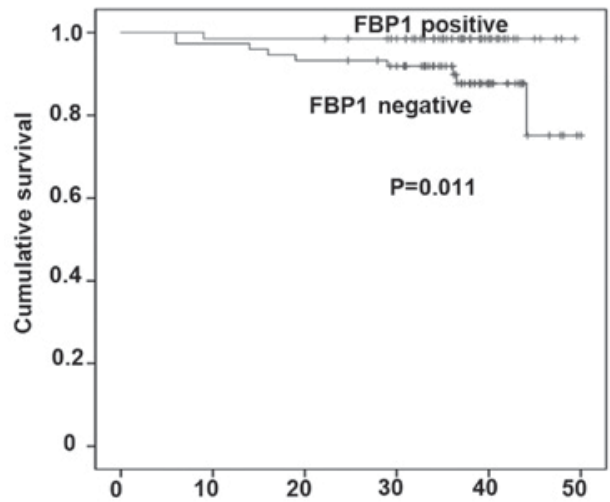

E

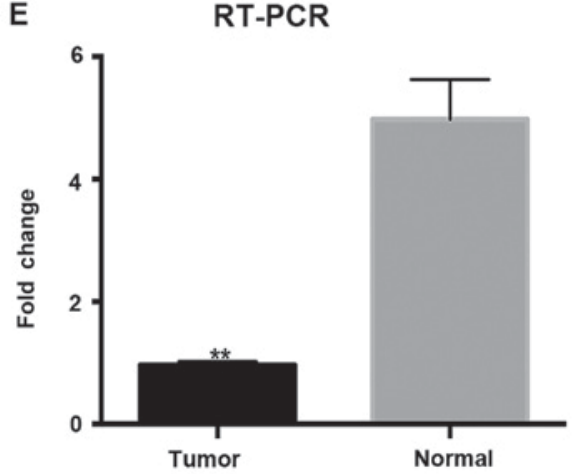

B

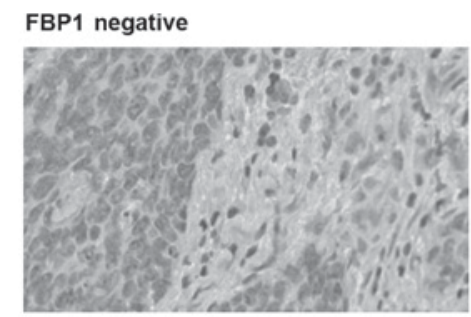

D

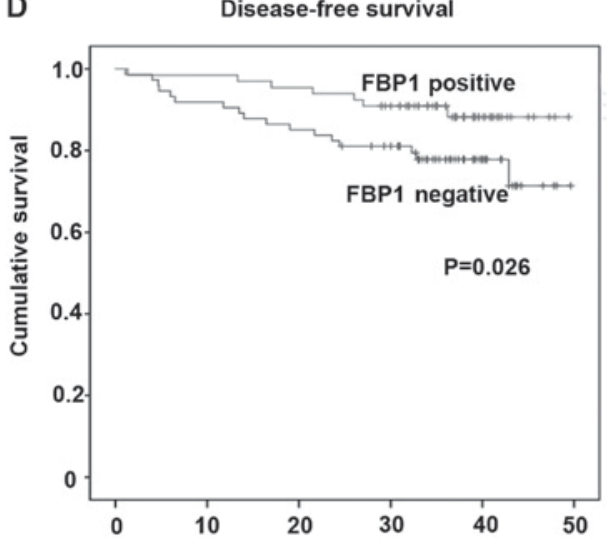

$\mathbf{F}$

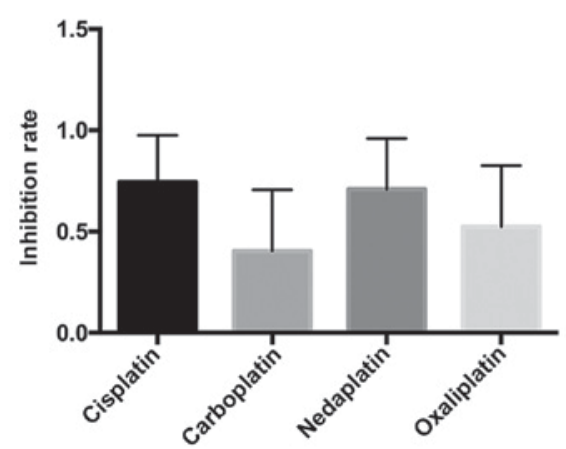

Figure 1. Decreased level of FBP1 is a negative prognostic factor in CSCC patients. (A) FBP1-positive expression and (B) FBP1-negative expression of tumor tissues were detected by immunohistochemistry (x400). Kaplan-Meier survival estimates showed that patients with FBP1-negative expression had a shorter (C) overall survival and (D) disease-free survival compared to those with FBP1-positive expression ( $\mathrm{P}=0.011$ and $\mathrm{P}=0.026$, respectively). (E) FBP1 mRNA expression level is lower in human CSCC tumor tissues than normal cervical tissues $(n=20, P=0.0005)$. (F) Inhibition rates of four platinum agents in CSCC patients. The median in vitro inhibition rates by cisplatin, carboplatin, nedaplatin, and oxaliplatin were $62,47,58$, and $52 \%$ respectively. FBP1, fructose-1,6-bisphosphatase-1; CSCC, squamous cell carcinoma of cervical cancer; RT-PCR, reverse transcription-polymerase chain reaction.

effects on the inhibition of tumor cells, and no significant difference in inhibition rates was observed between cisplatin and nedaplatin group (Fig. 1F). To further validate the effect of FBP1 on the chemosensitivity of cervical cancer cells, we examined the inhibition rate of HeLa and CaSki cells after cisplatin treatment when overexpressing FBP1 compared with negative control. The results showed that overexpression of FBP1 restored the cisplatin sensitivity of the cervical cancer cells (Fig. 3). However, by using the Kruskal-Wallis test, the FBP1 protein expression level showed no significant association with resistance to the four platinum agents in vitro (Table V). 
Table III. Various prognostic factors of CSCC.

\begin{tabular}{|c|c|c|c|}
\hline Prognostic factors & $\begin{array}{c}\text { FBP1- } \\
\text { negative }(\%)\end{array}$ & $\begin{array}{c}\text { FBP1- } \\
\text { positive }(\%)\end{array}$ & P-value ${ }^{a}$ \\
\hline Age, years & & & 0.176 \\
\hline$\leq 46$ (median) & 31 & 39 & \\
\hline$>46$ (median) & 39 & 31 & \\
\hline FIGO stage & & & $0.000^{c}$ \\
\hline IB & 25 & 45 & \\
\hline IIA & 39 & 25 & \\
\hline IIB & 6 & 0 & \\
\hline Tumor size $^{\mathrm{b}}, \mathrm{cm}$ & & & 0.390 \\
\hline$\leq 4$ & 44 & 39 & \\
\hline$>4$ & 26 & 31 & \\
\hline Pelvic LN & & & 0.150 \\
\hline Negative & 43 & 51 & \\
\hline Positive & 27 & 19 & \\
\hline LVSI & & & 0.080 \\
\hline Negative & 39 & 49 & \\
\hline Positive & 31 & 21 & \\
\hline $\begin{array}{l}\text { Depth of cervical } \\
\text { stromal invasion }\end{array}$ & & & 0.390 \\
\hline$\leq 2 / 3$ & 39 & 44 & \\
\hline$>2 / 3$ & 31 & 26 & \\
\hline Recurrence & & & 0.10 \\
\hline Local & 9 & 5 & \\
\hline Distant & 3 & 7 & \\
\hline $\begin{array}{l}\text { P16 expression } \\
\text { status }\end{array}$ & & & 1.000 \\
\hline Negative & 14 & 15 & \\
\hline Positive & 56 & 55 & \\
\hline
\end{tabular}

aTwo-sided $\chi^{2}$ test for distributions between negative and positive expression of FBP1. ${ }^{\text {b}}$ The largest tumor diameter after surgical resection. ${ }^{\mathrm{c}} \mathrm{P}<0.05$. CSCC, squamous cell carcinoma of cervical cancer; FBP1, fructose-1,6-bisphosphatase-1; FIGO, International Federation of Gynecology and Obstetrics; LN, lymph node; LVSI, lympho-vascular space invasion.

\section{Discussion}

Aerobic glycolysis, rather than oxidative phosphorylation, often occurs in tumor cells, which is called 'Warburg effect'. In this pathway, many metabolic intermediates were produced serving as biosynthesis molecules which are necessary for active cell proliferation. Moreover, the enhanced production of lactic acid from tumor cells will lower the $\mathrm{pH}$ of the microenvironment surrounding tumor cells, which can induce apoptosis for normal cells but not for tumor cells (17). In this study, the role of FBP1 which functions to antagonize glycolysis (8) in tumor tissues from CSCC patients was investigated. Our study revealed that the expression level of FBP1 was negatively correlated with the OS and DFS of cervical cancer patients. In addition, we found that the tumor tissues
Table IV. Clinical pathological features of 15 CSCC patients in reverse transcription-polymerase chain reaction analysis.

\begin{tabular}{|c|c|}
\hline Prognostic factors & Cases, $\mathrm{N}$ \\
\hline \multicolumn{2}{|l|}{ Age (years) } \\
\hline$\leq 43$ (median) & 10 \\
\hline$>43$ (median) & 10 \\
\hline \multicolumn{2}{|l|}{ FIGO stage } \\
\hline IB & 8 \\
\hline IIA & 8 \\
\hline IIB & 4 \\
\hline \multicolumn{2}{|l|}{ Tumor size $(\mathrm{cm})$} \\
\hline$\leq 4$ & 14 \\
\hline$>4$ & 6 \\
\hline \multicolumn{2}{|l|}{ Pelvic LN } \\
\hline Negative & 15 \\
\hline Positive & 5 \\
\hline \multicolumn{2}{|l|}{ LVSI } \\
\hline Negative & 16 \\
\hline Positive & 4 \\
\hline \multicolumn{2}{|c|}{ Depth of cervical stromal invasion } \\
\hline$\leq 2 / 3$ & 15 \\
\hline$>2 / 3$ & 5 \\
\hline \multicolumn{2}{|c|}{ P16 expression status } \\
\hline Negative & 3 \\
\hline Positive & 17 \\
\hline
\end{tabular}

CSCC, squamous cell carcinoma of cervical cancer; FIGO, International Federation of Gynecology and Obstetric; LN, lymph node; LVSI, lympho-vascular space invasion.

Table V. Association of FBP1 expression with resistance to the four platinum agents in vitro.

\begin{tabular}{lccc}
\hline & $\begin{array}{c}\text { In vitro tumor inhibition rates } \\
\text { (median } \pm \text { SE) }\end{array}$ & \\
\cline { 2 - 3 } $\begin{array}{l}\text { Platium } \\
\text { agents }\end{array}$ & $\begin{array}{c}\text { FBP1- } \\
\text { negative }\end{array}$ & $\begin{array}{c}\text { FBP1- } \\
\text { positive }\end{array}$ & P-value \\
\hline Cisplatin & $0.63 \pm 0.058$ & $0.61 \pm 0.059$ & 0.863 \\
Carboplatin & $0.49 \pm 0.060$ & $0.46 \pm 0.060$ & 0.737 \\
Nedaplatin & $0.57 \pm 0.059$ & $0.59 \pm 0.059$ & 0.865 \\
Oxaliplatin & $0.61 \pm 0.059$ & $0.43 \pm 0.060$ & 0.186 \\
\hline
\end{tabular}

${ }^{a}$ Kruskal-Wallis test for cisplatin, carboplatin, nedaplatin, and oxaliplatin between different distributions. FBP1, fructose-1,6-bisphosphatase-1; SE, standard error.

collected from CSCC patients expressed significantly lower level of FBP1 as compared with the normal cervical tissues. Our data clearly indicated that FBP1 appears to be functional tumor suppressor involved in cervical carcinogenesis, which could be used as a biomarker for CSCC. Fella et al (18) also 
A

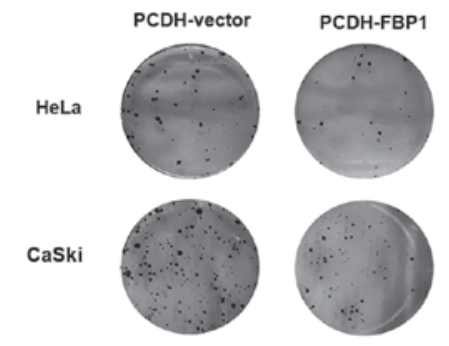

C

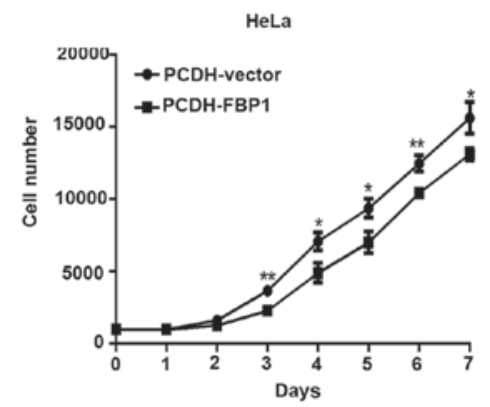

E

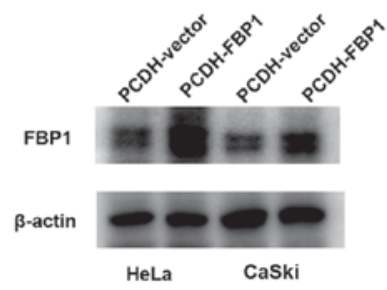

B

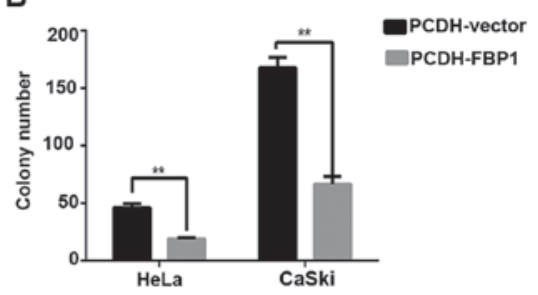

D Caski
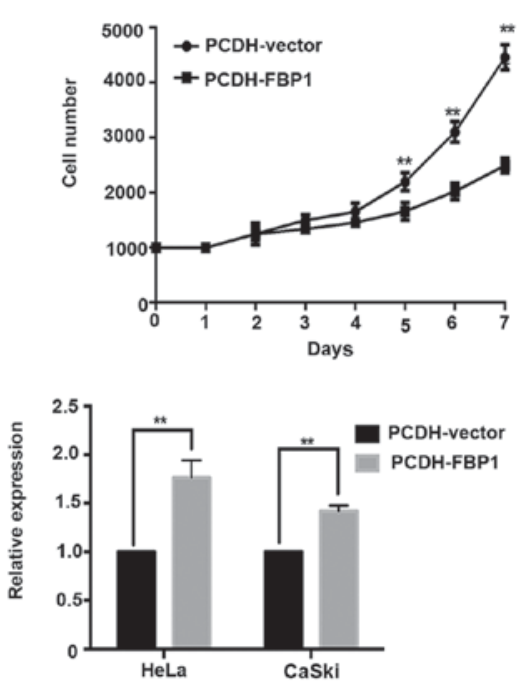
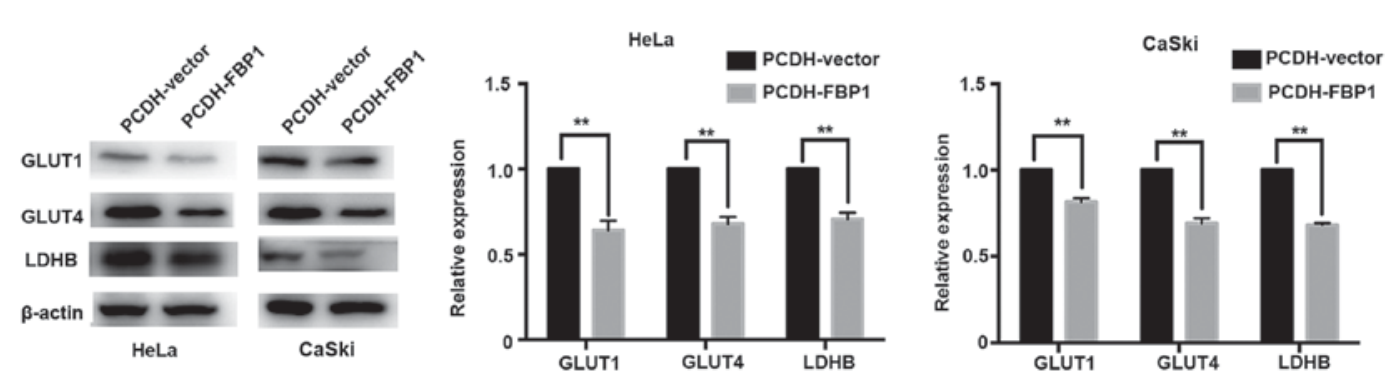

Figure 2. Induction of FBP1 on colony formation, and cell proliferation ability in cervical cancer cell lines. Upregulation of FBP1 suppressed colony formation of HeLa PCDH-FBP1 cells (A) and CaSki PCDH-FBP1 cells (B) compared to PCDH-vector cells. Overexpression of FBP1 suppressed cell proliferation of HeLa PCDH-FBP1 cells (C) and CaSki PCDH-FBP1 cells (D) compared to PCDH-vector cells. Established cervical cancer cell lines with FBP1 overexpression was confirmed by western blot analysis. The expression levels of GLUT1, GLUT4 and LDHB proteins were lower in HeLa or CaSki cells PCDH-FBP1 compared with PCDH-vector cells (E). Data are means of three independent experiments $\pm \mathrm{SEM} .{ }^{*} \mathrm{P}<0.05,{ }^{* *} \mathrm{P}<0.01$. FBP1, fructose-1,6-bisphosphatase-1; GLUT, glucose transporter; LDHB, lactate dehydrogenase B.

A

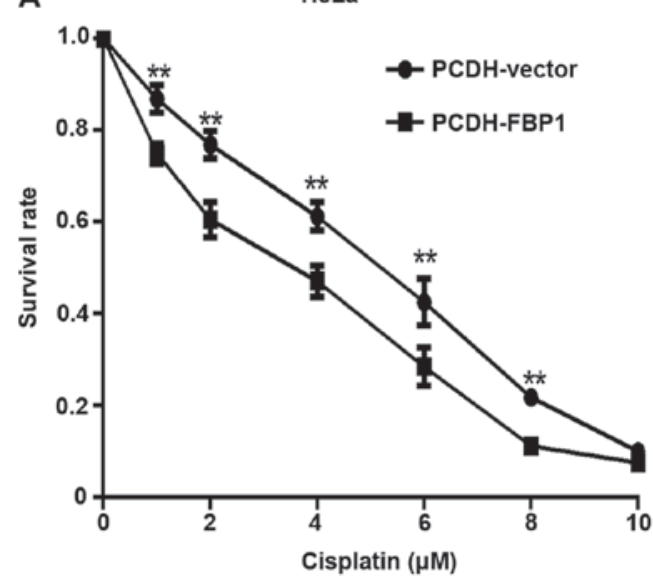

B

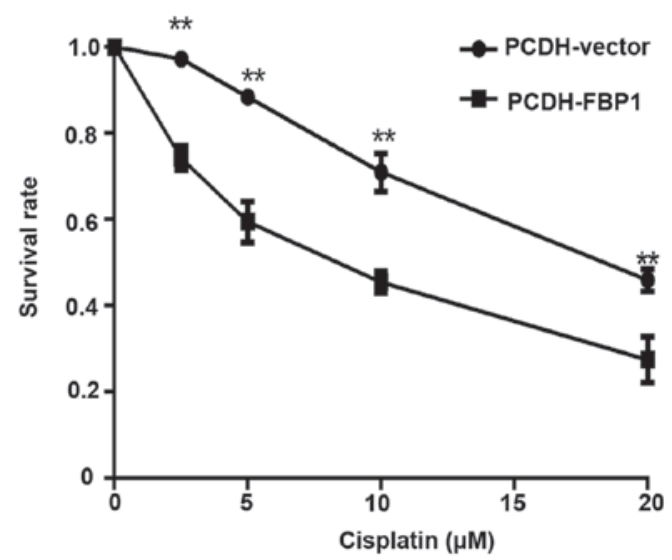

Figure 3. FBP1 overexpression sensitizes cervical cancer cells to cisplatin. Enhancement of FBP1 confered cervical cancer cells (A) HeLa and (B) CaSki more sensitive to the cytotoxic effect of cisplatin compared to PCDH-vector cells. Data are means of three independent experiments \pm standard error. ${ }^{* *} \mathrm{P}<0.01$. FBP1, fructose-1,6-bisphosphatase-1. 
investigated that FBPase protein is very early downregulated and postulated it as an early predictive biomarker for liver carcinogenicity.

Although the exact mechanisms of FBP1 down regulation in carcinogenesis is unclear, studies of several solid tumors, such as liver, colon, gastric as well as breast cancer cells showed an increased methylation of FBP1 promoter $(8,9,19)$. Since epigenetic modification are known to contribute to the multitude of metabolic changes in cancer cells (20), during carcinogenesis the genome simultaneously undergoes genome-wide hypomethylation and regional hypermethylation of $\mathrm{CpG}$ islands, which may be of selective advantage for the incipient tumor cells (21). Inhibition of $\mathrm{NF}-\kappa \mathrm{B}$ restored $\mathrm{FBP} 1$ expression in gastric cancer cells, partially through demethylation of FBP1 promoter (8). In breast cancer, Snail-G9a-Dnmt1 complex, which is critical for E-cadherin promoter silencing, is also required for the promoter methylation of FBP1 in basal-like breast cancer (BLBC) (22). With promoter methylation, downregulated expression of FBP1 confers to enhancing 'Warburg effect' by increasing glucose uptake, lactate secretion and glycolytic intermediates for biosynthesis in mitochondria of tumor cells. And this glycolytic switch could help tumor cells, rather than normal cells acquire more energy in the process of carcinogenesis and progression. Besides, loss of FBP1 might promote carcinogenesis in a catalytic activity independent way, by decreasing interaction with hypoxia inducible factor (HIF) domain directly to promote cell proliferation or by increasing the number of cancer stem cells which contribute to tumor recurrence and therapeutic resistance $(10,17,19,22)$. Our study showed that glycolysis-related protein expression was significantly inhibited by enhancing FBP1 expression. And overexpression of FBP1 could further inhibit the colony formation and proliferation ability of cervical cancer cells. These results indicated that FBP1 might inhibit the growth of cervical cancer cells partially through suppressing glycolytic pathway.

Chemotherapy has been widely used in the treatment of various cancers, including advanced or recurrent and/or metastatic cervical cancer $(2-4,23,24)$. Platinum especially cisplatin is still the basic agent in the chemotherapeutic regimen for CSCC patients $(25,26)$. However, frequent resistance to platinum agents in cervical cancer cells obstructs the progress in satisfying treatment. In this study, we detected in vitro inhibition rate of tumor cells of four platinum agents including cisplatin, carboplatin, nedaplatin, and oxaliplatin. We found the inhibition rates of nedaplatin and cisplatin were much higher than that of carboplatin and oxaliplatin, which explained why cisplatin drugs are most commonly used in chemotherapy of CSCC patients (27). Although we did not detect the statistical significant association of in vitro tumor inhibition rates with FBP1 expression, FBP1-negative tumor cells had a lower inhibition rates in most of the platinum treated groups, compared with FBP1-negative tumor cells. Several studies revealed that increased expression of glycolytic pathway associated genes, such as HIF-1 $\alpha$, GLUT1, and lactate dehydrogenase (LDH) were also associated with drug resistance in cancer $(28,29)$, and our data showed that overexpression of FBP1 restored the sensitivity to cisplatin and suppressed glycolysis-related protein GLUT1, GLUT4 and LDHB in cervical cancer cells. Collectively, these results suggested that FBP1 might block the glucose metabolism to further impact the chemosensitivity of cervical cancer cells. However, further studies are necessary to reveal the mechanisms of this phenomenon.

In conclusion, we have found for the first time that FBP1 expression had an inverse correlation with prognosis of CSCC patients. And decreased level of FBP1 could promote carcinogenesis of CSCC patients. Furthermore, FBP1 overexpression could inhibit proliferation ability and restore chemosensitivity of cervical cancer cells probably by suppressing glycolysis pathway. These findings suggested that FBP1 could be a useful biomarker for predicting prognosis of CSCC patients, and restoring expression of FBP1 could be a potential therapeutic target to suppress tumor progression. However, there are some limitations in our study. Firstly, we did not perform in vivo experiments to further confirm our hypothesis. Secondly, a deeper investigation is needed to clarify potential mechanisms through which decreased level of FBP1 promotes carcinogenesis and chemoresistance in CSCC. Indeed, our study is exploratory and descriptive, we would try our best to solve these problems in the next future.

\section{Acknowledgements}

The present study was supported by National Nature Science Foundation of China (NSFC1212) (grant no. 81202050) for Xi Cheng. We would like to thank Guangqi Qin and Xu Cai of Fudan University Shanghai Cancer Center for performing tissue microarray and immunohistochemistry techniques, respectively.

\section{References}

1. Torre LA, Bray F, Siegel RL, Ferlay J, Lortet-Tieulent J and Jemal A: Global cancer statistics, 2012. CA Cancer J Clin 65: 87-108, 2015.

2. Lissoni AA, Colombo N, Pellegrino A, Parma G, Zola P, Katsaros D, Chiari S, Buda A, Landoni F, Peiretti M, et al: A phase II, randomized trial of neo-adjuvant chemotherapy comparing a three-drug combination of paclitaxel, ifosfamide and cisplatin (TIP) versus paclitaxel and cisplatin (TP) followed by radical surgery in patients with locally advanced squamous cell cervical carcinoma: The snap-02 Italian collaborative study. Ann Oncol 20: 660-665, 2009.

3. Katanyoo K, Tangjitgamol S, Chongthanakorn M, Tantivatana T, Manusirivithaya S, Rongsriyam K and Cholpaisal A: Treatment outcomes of concurrent weekly carboplatin with radiation therapy in locally advanced cervical cancer patients. Gynecol Oncol 123: 571-576, 2011

4. Symonds RP, Gourley C, Davidson S, Carty K, McCartney E, Rai D, Banerjee S, Jackson D, Lord R, McCormack M, et al: Cediranib combined with carboplatin and paclitaxel in patients with metastatic or recurrent cervical cancer (CIRCCa): A randomised, double-blind, placebo-controlled phase 2 trial. Lancet Oncol 16: 1515-1524, 2015.

5. Monk BJ and Tewari KS: Evidence-based therapy for recurrent cervical cancer. J Clin Oncol 32: 2687-2690, 2014.

6. Monk BJ, Sill MW, McMeekin DS, Cohn DE, Ramondetta LM, Boardman CH, Benda J and Cella D: Phase III trial of four cisplatin-containing doublet combinations in stage IVB, recurrent, or persistent cervical carcinoma: A Gynecologic Oncology Group Study. J Clin Oncol 27: 4649-4655, 2009.

7. Tillmann $\mathrm{H}$ and Eschrich $\mathrm{K}$ : Isolation and characterization of an allelic cDNA for human muscle fructose-1,6-bisphosphatase. Gene 212: 295-304, 1998.

8. Liu X, Wang X, Zhang J, Lam EK, Shin VY, Cheng AS, Yu J, Chan FK, Sung JJ and Jin HC: Warburg effect revisited: An epigenetic link between glycolysis and gastric carcinogenesis. Oncogene 29: 442-450, 2010.

9. Chen M, Zhang J, Li N, Qian Z, Zhu M, Li Q, Zheng J, Wang X and Shi G: Promoter hypermethylation mediated downregulation of FBP1 in human hepatocellular carcinoma and colon cancer. PLoS One 6: e25564, 2011. 
10. Li B, Qiu B, Lee DS, Walton ZE, Ochocki JD, Mathew LK, Mancuso A, Gade TP, Keith B, Nissim I and Simon MC: Fructose-1,6-bisphosphatase opposes renal carcinoma progression. Nature 513: 251-255, 2014.

11. Bigl M, Jandrig B, Horn LC and Eschrich K: Aberrant methylation of human L- and M-fructose 1,6-bisphosphatase genes in cancer. Biochem Biophys Res Commun 377: 720-724, 2008.

12. Vander Heiden MG, Cantley LC and Thompson CB: Understanding the warburg effect: The metabolic requirements of cell proliferation. Science 324: 1029-1033, 2009.

13. DeBerardinis RJ and Thompson CB: Cellular metabolism and disease: What do metabolic outliers teach us? Cell 148: 1132-1144, 2012.

14. Cheng X, Cai SM, Li ZT, Wu XH, Ding YQ, Wang XE and Zang RY: Concurrent chemotherapy and adjuvant extended field irradiation after radical surgery for cervical cancer patients with lymph node metastases. Int J Gynecol Cancer 18: 779-784, 2008.

15. Shi TY, Yang G, Tu XY, Yang JM, Qian J, Wu XH, Zhou XY, Cheng X and Wei Q: RAD52 variants predict platinum resistance and prognosis of cervical cancer. PLoS One 7: e50461, 2012.

16. Cheng X, Yang G, Schmeler KM, Coleman RL, Tu X, Liu J and Kavanagh JJ: Recurrence patterns and prognosis of endometrial stromal sarcoma and the potential of tyrosine kinase-inhibiting therapy. Gynecol Oncol 121: 323-327, 2011.

17. Vaupel P, Kallinowski F and Okunieff P: Blood flow, oxygen and nutrient supply, and metabolic microenvironment of human tumors: A review. Cancer Res 49: 6449-6465, 1989.

18. Fella K, Glückmann M, Hellmann J, Karas M, Kramer PJ and Kröger M: Use of two-dimensional gel electrophoresis in predictive toxicology: Identification of potential early protein biomarkers in chemically induced hepatocarcinogenesis. Proteomics 5: 1914-1927, 2005.

19. Hirata H, Sugimachi K, Komatsu H, Ueda M, Masuda T, Uchi R, Sakimura S, Nambara S, Saito T, Shinden Y, et al: Decreased expression of fructose-1,6-bisphosphatase associates with glucose metabolism and tumor progression in hepatocellular carcinoma. Cancer Res 76: 3265-3276, 2016.

20. Saito Y, Liang G, Egger G, Friedman JM, Chuang JC, Coetzee GA and Jones PA: Specific activation of microRNA-127 with downregulation of the proto-oncogene BCL6 by chromatin-modifying drugs in human cancer cells. Cancer Cell 9: 435-443, 2006.

21. Jones PA and Baylin SB: The fundamental role of epigenetic events in cancer. Nat Rev Genet 3: 415-428, 2002.
22. Dong C, Yuan T, Wu Y, Wang Y, Fan TW, Miriyala S, Lin Y, Yao J, Shi J, Kang T, et al: Loss of FBP1 by snail-mediated repression provides metabolic advantages in basal-like breast cancer. Cancer Cell 23: 316-331, 2013.

23. Green JA, Kirwan JM, Tierney JF, Symonds P, Fresco L, Collingwood $\mathrm{M}$ and Williams CJ: Survival and recurrence after concomitant chemotherapy and radiotherapy for cancer of the uterine cervix: A systematic review and meta-analysis. Lancet 358: 781-786, 2001.

24. Zuliani AC, Esteves SC, Teixeira LC, Teixeira JC, de Souza GA and Sarian LO: Concomitant cisplatin plus radiotherapy and high-dose-rate brachytherapy versus radiotherapy alone for stage IIIB epidermoid cervical cancer: A randomized controlled trial. J Clin Oncol 32: 542-547, 2014.

25. Kitagawa R, Katsumata N, Shibata T, Kamura T, Kasamatsu T, Nakanishi T, Nishimura S, Ushijima K, Takano M, Satoh T and Yoshikawa H: Paclitaxel plus carboplatin versus paclitaxel plus cisplatin in metastatic or recurrent cervical cancer: The open-label randomized phase III trial JCOG0505. J Clin Oncol 33: 2129-2135, 2015.

26. Mabuchi S, Isohashi F, Yokoi T, Takemura M, Yoshino K, Shiki Y, Ito K, Enomoto T, Ogawa K and Kimura T: A phase II study of postoperative concurrent carboplatin and paclitaxel combined with intensity-modulated pelvic radiotherapy followed by consolidation chemotherapy in surgically treated cervical cancer patients with positive pelvic lymph nodes. Gynecol Oncol 141: 240-246, 2016.

27. Dunst $\mathbf{J}$ and Haensgen G: Simultaneous radiochemotherapy in cervical cancer: Recommendations for chemotherapy. Strahlenther Onkol 177: 635-640, 2001.

28. Sowa T, Menju T, Chen-Yoshikawa TF, Takahashi K, Nishikawa S, Nakanishi T, Shikuma K, Motoyama H, Hijiya K, Aoyama A, et al: Hypoxia-inducible factor 1 promotes chemoresistance of lung cancer by inducing carbonic anhydrase IX expression. Cancer Med 6: 288-297, 2017

29. Song K, Li M, Xu X, Xuan Li, Huang G and Liu Q: Resistance to chemotherapy is associated with altered glucose metabolism in acute myeloid leukemia. Oncol Lett 12: 334-342, 2016. 